\title{
Parallel electric field amplification by phase mixing of Alfven waves
}

\author{
N. H. Bian and E. P. Kontar
}

Department of Physics \& Astronomy, University of Glasgow, G12 8QQ, UK

e-mail: nbian@astro.gla.ac.uk

Received 13 July 2010 / Accepted 10 November 2010

\section{ABSTRACT}

\begin{abstract}
Context. Several numerical studies have identified phase mixing of low-frequency Alfven waves as a means of parallel electric field amplification and acceleration of electrons in a collisionless plasma.

Aims. Theoretical explanations are given of how phase mixing amplifies the parallel electric field and, as a consequence, also leads to enhanced collisionless damping of the wave by energy transfer to the electrons.

Methods. Our results are based on the properties of the Alfven waves in a warm plasma. These results are obtained within the framework of drift-kinetic theory.

Results. Phase mixing in a collisionless low- $\beta$ plasma proceeds in a manner very similar to the resistive case, except that electron Landau damping is the primary energy dissipation channel. The time and length scales involved are evaluated. We also focus on the evolution of the parallel electric field and calculate its maximum value in the course of its amplification
\end{abstract}

Key words. magnetohydrodynamics (MHD) - waves - Sun: corona

\section{Introduction}

At finite wave numbers in the direction perpendicular to the ambient magnetic field, Alfven waves produce a compression of the plasma that results in the creation of a parallel electric field via the thermo-electric effect, i.e. from electron pressure fluctuations along the magnetic field lines (see discussion below and, e.g., Hollweg 1999). This situation occurs in a plasma with a pressure parameter $\beta$ that is greater than $m_{\mathrm{e}} / m_{\mathrm{i}}$. In contrast, when the pressure parameter is below $m_{\mathrm{e}} / m_{\mathrm{i}}$, the parallel electric field of the Alfven wave is mainly balanced by electron inertia. These warm and cold plasma regimes of the dispersive Alfven wave are dubbed kinetic and inertial, respectively. This parallel electric field, whose magnitude increases with $k_{\perp}$, leads to wave-particle interactions, hence to Landau damping of the Alfven wave.

The importance of this parallel electric field was pointed out some time ago by Hasegawa \& Chen (1976). Indeed, they consider resonant absorption (Hasegawa \& Chen 1974) in a warm plasma and argue that it is a manifestation of mode conversion from the MHD Alfven wave (AW) to the kinetic Alfven wave (KAW). As a result, the physical mechanism of the heating depends on the collisionless absorption of the KAW. Although the original motivation was to explain electron heating in laboratory fusion plasmas, this electric field was also proposed as a mechanism that can accelerate electrons in space plasmas (Hasegawa 1976; Hasegawa \& Mima 1978; Hasegawa 1985; Goertz \& Boswell 1979) and that helps for understanding solar coronal heating (Ionson 1978).

Heyvaerts \& Priest (1983) also introduced the idea of phase mixing to improve the efficiency of AW dissipation. Their theory is based on visco-resistive magnetohydrodynamics (MHD). Since then, MHD phase mixing has attracted a significant amount of attention in the context of heating open magnetic structures in the solar corona (Parker 1991; Nakariakov et al. 1997; Botha et al. 2000; De Moortel et al. 2000; Hood et al. 2002). Popular excitation mechanisms for coronal AWs in open magnetic structures are photospheric motions for the low-frequency and chromospheric reconnection events for the high-frequency range of the spectrum.

Phase mixing can be understood as the refraction of the wave while it propagates along a magnetic field with transverse variation in the Alfven velocity, i.e. the progressive increase of its $k_{\perp}$. This is a special occurrence of an anisotropic conservative energy cascade, a phenomenon generally attributed to non-linear interactions between wave packets. For more details, see the discussion in (Bian \& Tsiklauri 2008). Therefore, it is not surprising that phase mixing produces amplification of the parallel electric field that accompanies the Alfven wave in a collisionless plasma, although this cannot be understood within the framework of ideal MHD theory, which assumes $E_{\|}=0$.

Previous numerical studies of phase mixing in a collisionless plasma have identified its involvement in the generation of a parallel electric field and acceleration of electrons (Tsiklauri et al. 2005a,b; Tsiklauri \& Haruki 2008); see also Genot et al. (1999, 2004) in the context of the magnetosphere. As stated above, the same features have been established already some time ago by Hasegawa and Chen for resonant absorption. Here, we provide a detailed discussion of the role played by phase mixing in both parallel electric field amplification and enhanced electron Landau damping of AWs in a collisionless plasma.

The calculations are based on the drift-kinetic theory presented in Sect. 2, which is valid in the limit of low-frequency fluctuations with $\omega \ll \omega_{\mathrm{ci}}, \omega_{\mathrm{ci}}$ is the ion cyclotron frequency. Phase mixing and enhanced electron Landau damping of AWs in a collisionless low- $\beta$ plasma are considered in Sect. 3. Parallel electric field amplification is studied in Sect. 4. Conclusions and discussions are provided in Sect. 5.

\section{Kinetic properties of the Alfven wave in a warm collisionless plasma}

Our starting point is the linearized drift-kinetic equation describing the magnetic field aligned dynamics of the electrons:

$$
\partial_{t} f_{1}+v_{\|} \nabla_{\|} f_{1}-\frac{e}{m_{\mathrm{e}}} E_{\|} \partial_{v_{\|}} f_{0}=0 .
$$


Here, the electron distribution is written as the sum of a background and a small perturbation; i.e., $f\left(x, y, z, v_{\|}, t\right)=f_{0}\left(v_{\|}\right)+$ $f_{1}\left(x, y, z, v_{\|}, t\right)$. We assume the existence of a background magnetic field $B_{0}=B_{0} z$ directed along $z$. The parallel component of any field $F$ is written as $F_{\|}$, and the perpendicular component is $F_{\perp}$. This notation also holds for the differential operator $\nabla$, hence the notation $k_{\perp}$ and $k_{\|}$for the perpendicular and the parallel wave number, respectively.

The drift-kinetic equation is supplemented by Maxwell's equations. Faraday's law is

$$
E_{\|}=-\nabla_{\|} \phi-\frac{1}{c} \frac{\partial A_{\|}}{\partial t}
$$

where $\phi$ is the electric potential, $A_{\|}$the parallel component of the vector potential. The parallel component of Ampere's law reads

$$
\nabla_{\perp}^{2} A_{\|}=\frac{4 \pi e}{c} \int v_{\|} f_{1} \mathrm{~d} v_{\|} \cdot
$$

The above system is closed by the quasi-neutrality condition, which in the limit $k_{\perp} \rho_{\mathrm{i}} \ll 1$, reads as

$$
n_{0} \rho_{\mathrm{i}}^{2} \nabla_{\perp}^{2} \frac{e \phi}{T_{0 \mathrm{i}}}=\int f_{1} \mathrm{~d} v_{\|},
$$

where $\rho_{\mathrm{i}}$ is the thermal ion Larmor radius at the temperature $T_{0 \mathrm{i}}$, and $n_{0}$ the background density. We set the Boltzmann constant to unity, which means that the temperature has the unit of energy. While this so-called gyrokinetic Poisson equation (Eq. (4)) includes the effect associated with the ion dynamics, i.e. their perpendicular polarization drift, the electron response along the perturbed field lines is described by the drift-kinetic equation (Eq. (1)).

We assume a small deviation $f_{1}$ from an equilibrium Maxwellian distribution $f_{0}$ :

$$
f_{0}\left(v_{\|}\right)=\frac{n_{0}}{\sqrt{\pi} v_{\mathrm{te}}} \mathrm{e}^{-v_{\|}^{2} / v_{\mathrm{te}}^{2}},
$$

with $v_{\text {te }}$ the electron thermal speed. The above closed set of equations is a self-consistent description of the linear plasma dynamics and is a simple form of gyrokinetics. This description of the plasma dynamics is based on an averaging of the kinetic and Maxwell equations over the gyromotion of the particles. This procedure is valid in the limit of frequencies that are small compared to the ion cyclotron frequency and within the limit of a small Larmor radius. Moreover, it is assumed that fluctuations are small and anisotropic: $k_{\|} / k_{\perp} \sim \delta B / B_{0} \ll 1$. As for reduced MHD, there is pressure balance in the direction perpendicular to the background magnetic field, such that the fast mode is ordered out. Finally, because the gyroaverage procedure eliminates the cyclotron resonance, the only type of wave-particle interaction that remains possible is through the Landau resonance between the particles and the parallel electric force. We refer to the recent work by Schekochihin et al. (2009) for a review on astrophysical gyrokinetics.

The electron density perturbation is defined as $n_{\mathrm{e}}=\int f_{1} \mathrm{~d} v_{\|}$ and the parallel current perturbation as $J_{\|}=-e \int v_{\|} f_{1} \mathrm{~d} v_{\|}=$ $-e n_{0} u_{\| \mathrm{e}}$, where $u_{\| \mathrm{e}}$ is the electron parallel velocity, and the electron pressure perturbation is defined as $P_{\mathrm{e}}=m_{\mathrm{e}} \int v_{\|}^{2} f_{1} \mathrm{~d} v_{z}$. Ampere's law and Poisson law can thus be written, respectively, as $\nabla_{\perp}^{2} A_{\|}=-(4 / \pi c) J_{\|}$and $\rho_{\mathrm{i}}^{2} \nabla_{\perp}^{2} e \phi / T_{0 \mathrm{i}}=n_{\mathrm{e}} / n_{0}$. On one hand, taking the zeroth order moment of the electron kinetic equation provides the electron continuity equation:

$$
\frac{\partial n_{\mathrm{e}}}{\partial t}+n_{0} \mathrm{i} k_{\|} u_{\| \mathrm{e}}=0
$$

On the other hand, the first moment provides the parallel electron momentum equation:

$$
n_{0} m_{\mathrm{e}} \frac{\partial u_{\| \mathrm{e}}}{\partial t}=-\mathrm{i} k_{\|} P_{\mathrm{e}}-n_{0} e E_{\|} .
$$

It is usual to refer to the last equation as Ohms's law, and $P_{\mathrm{e}}=$ $n_{\mathrm{e}} T_{0 \mathrm{e}}$ for an isothermal plasma. Therefore, there are two possible sources of parallel electric field associated with the electron dynamics: inertia and pressure (or density) variations along the field lines. The continuity equation combined with Poisson law yields a vorticity equation :

$$
\frac{\partial}{\partial t} \rho_{\mathrm{i}}^{2} \nabla_{\perp}^{2} \frac{e \phi}{T_{0 \mathrm{i}}}+\frac{c}{4 \pi e n_{0}} \mathrm{i} k_{\|} \nabla_{\perp}^{2} A_{\|}=0
$$

Neglecting first the effects of electron inertia and electron pressure gradient in Ohm's law yields the MHD Ohm's law $E_{\|}=0$, i.e.,

$$
\frac{1}{c} \frac{\partial A_{\|}}{\partial t}=-\mathrm{i} k_{\|} \phi
$$

Introducing the stream and flux function for the velocity $\boldsymbol{u}_{\perp}=$ $z \times \nabla_{\perp} \varphi$, and the magnetic field $\boldsymbol{B}_{\perp} / \sqrt{4 \pi n_{0} m_{\mathrm{i}}}=z \times \nabla_{\perp} \psi$, defined as $\varphi=\left(c / B_{0}\right) \phi$ and $\psi=-A_{\|} / \sqrt{4 \pi n m_{i}}$, gives

$$
\begin{aligned}
& \frac{\partial}{\partial t} \nabla_{\perp}^{2} \varphi=v_{\mathrm{A}} \mathrm{i} k_{\|} \nabla_{\perp} \psi, \\
& \frac{\partial \psi}{\partial t}=v_{\mathrm{A}} \mathrm{i} k_{\|} \varphi
\end{aligned}
$$

with $v_{\mathrm{A}}=B_{0} / \sqrt{4 \pi n_{0} m_{\mathrm{i}}}$ the Alfven velocity. These two equations are the standard linearized reduced-MHD equations describing shear-Alfven waves with frequency:

$$
\omega= \pm v_{\mathrm{A}} k_{\|} \text {. }
$$

When the parallel electric field is produced by a density fluctuation in Ohm's law, we have $E_{\|}=-\mathrm{i} k_{\|} T_{0 \mathrm{e}}\left(n_{\mathrm{e}} / n_{0}\right)$. Using the Poisson equation, $E_{\|}=-\mathrm{i} k_{\|} \rho_{\mathrm{s}}^{2} \nabla_{\perp}^{2} \phi$, which also reveals the vortical nature of the parallel electric field. The parameter $\rho_{\mathrm{s}}=$ $c_{\mathrm{s}} / \omega_{c i}=\sqrt{T_{0 \mathrm{e}} / T_{0 \mathrm{i}}} \rho_{\mathrm{i}}$ is the ion gyroradius at the electron temperature. By including this parallel electric field in Ohm's law, an extension of the previous reduced-MHD system now takes the form

$$
\begin{aligned}
& \frac{\partial}{\partial t} \nabla_{\perp}^{2} \varphi=v_{\mathrm{A}} \mathrm{i} k_{\|} \nabla_{\perp} \psi, \\
& \frac{\partial \psi}{\partial t}=v_{\mathrm{A}} \mathrm{i} k_{\|}\left(\varphi-\rho_{\mathrm{s}}^{2} \nabla_{\perp}^{2} \varphi\right),
\end{aligned}
$$

which describes the dynamics of kinetic Alfven waves with frequency

$$
\omega= \pm v_{\mathrm{A}} k_{\|} \sqrt{1+\rho_{\mathrm{s}}^{2} k_{\perp}^{2}}
$$

It is worth noticing that Eqs. (13), (14) can also be obtained directly from two-fluid MHD theory by retaining the Hall and electron pressure effects in Ohm's law (Bian \& Tsiklauri 2009). Using the above results, it is easily seen that, for kinetic Alfven waves, the magnitude of the parallel electric field is related to $B_{\perp}$ by

$$
E_{\|}=\frac{v_{\mathrm{A}}}{c} k_{\|} \frac{k_{\perp} \rho_{\mathrm{s}}^{2}}{\sqrt{1+k_{\perp}^{2} \rho_{\mathrm{s}}^{2}}} B_{\perp} .
$$


The above fluid derivation of the Alfven wave frequency gives the same result as its kinetic counterpart, however the latter, which is presented below, is more complete in the sense that it also provides the imaginary part associated with Landau damping. The electron kinetic equation can be solved for the perturbed distribution function $f_{1}$, i.e.,

$$
f_{1}=\mathrm{i} \frac{e}{m_{\mathrm{e}}} E_{\|} \frac{2 n_{0}}{\sqrt{\pi} k_{\|} v_{\mathrm{te}}^{3}} \frac{v_{\|} / v_{\mathrm{te}}}{v_{\|} / v_{\mathrm{te}}-\omega / k_{\|} v_{\mathrm{te}}} \mathrm{e}^{-v_{\|}^{2} / v_{\mathrm{te}}^{2}} .
$$

Some notations are introduced: $x=v_{\|} / v_{\mathrm{te}}, \alpha=\omega / k_{\|} v_{\mathrm{te}}$ and

$$
Z_{n}(\alpha)=\frac{1}{\sqrt{\pi}} \int \frac{x^{n}}{x-\alpha} \mathrm{e}^{-x^{2}} \mathrm{~d} x,
$$

with $Z_{0}(\alpha)$ the standard plasma dispersion function. We also summarize some properties of the functions $Z_{n}: Z_{1}=1+\alpha Z_{0}$, $Z_{2}=\alpha Z_{1}$. Moreover, in the limit $\alpha \ll 1$

$$
Z_{0}(\alpha) \sim-2 \alpha+\mathrm{i} \sqrt{\pi}\left(1-\alpha^{2}\right) .
$$

Using the above properties, it follows that the density and current perturbations are related to the parallel electric field through

$$
\int f_{1} \mathrm{~d} v_{\|}=\frac{2 \mathrm{i} e n_{0}}{m_{\mathrm{e}} k_{\|} v_{\mathrm{te}}^{2}}\left[1+\alpha Z_{0}(\alpha)\right] E_{\|},
$$

for the density, and

$$
\int f_{1} v_{\|} \mathrm{d} v_{\|}=\frac{2 \mathrm{i} e n_{0} \omega}{m_{\mathrm{e}} k_{\|}^{2} v_{\mathrm{te}}^{2}}\left[1+\alpha Z_{0}(\alpha)\right] E_{\|} .
$$

As a result, the relation between the parallel current and the parallel electric field is

$$
J_{\|}=\frac{-\mathrm{i} \omega}{4 \pi k_{\|}^{2} \lambda_{\mathrm{De}}^{2}}\left[1+\alpha Z_{0}(\alpha)\right] E_{\|} .
$$

It is convenient to define a collisionless plasma conductivity $\sigma$ as

$$
J_{\|}=\sigma E_{\|} .
$$

Its imaginary part results in the dispersion of the Alfven wave and its real part yields the collisionless dissipation. In the limit $\alpha \equiv \omega / k_{\|} v_{\mathrm{te}} \ll 1$, the real part is

$$
\sigma_{r} \simeq \frac{\mathrm{e}^{2} m_{\mathrm{e}}^{1 / 2} n_{0} \omega^{2}}{k_{\|}^{3} T_{0 \mathrm{e}}^{3 / 2}}
$$

This also gives the energy per united time transferred to the electrons through the relation

$$
Q=\operatorname{Re}\left(J_{\|} E_{\|}^{*}\right)
$$

i.e.,

$$
Q=\frac{\sqrt{\pi} \omega^{2}}{k_{\|}^{3} \lambda_{D e}^{2} v_{\mathrm{te}}} U_{E_{\|}}
$$

with $\lambda_{\text {De }}$ the electron Debye length and $U_{E_{\|}}=\left|E_{\|}^{2}\right| / 8 \pi$ the energy density of the parallel component of the electric field. It is, in fact, a standard result that the asymptotic $\omega t \gg 1$ averaged power transferred to electrons, $Q=\int v_{\|}\left\langle-e E_{\|} f_{1}\right\rangle \mathrm{d} v_{\|}$, due to the presence of an harmonic electric field fluctuation $E_{\|}=\cos \left(k_{\|} z-\right.$ $\omega t$ ), is

$$
Q=-\pi \frac{\mathrm{e}^{2} E_{\|}^{2}}{2 m_{\mathrm{e}} k_{\|}}\left[v_{\|} \frac{\partial f_{0}}{\partial v_{\|}}\right]_{v_{\|}=\omega / k_{\|}} .
$$

It can be verified easily from Eq. (1) and for a Maxwellian distribution that this last result is equivalent to Eq. (26). Using the relation between $E_{\|}$and $B_{\perp}, Q$ can finally be expressed in term of the magnetic energy, $U_{\mathrm{B}}=\left|B_{\perp}^{2}\right| / 8 \pi$,

$$
Q=\frac{\sqrt{\pi} \omega^{2}}{k_{\|} v_{\mathrm{te}}} \frac{k_{\perp}^{2} \rho_{\mathrm{s}}^{2}}{1+k_{\perp}^{2} \rho_{\mathrm{s}}^{2}} U_{\mathrm{B}} .
$$

The coefficient of proportionality between $Q$ and $U_{\mathrm{B}}$, which has the dimension of the inverse of a time, is nothing else than the Landau damping rate.

The Landau damping rate is now obtained directly from the complex dispersion relation. The kinetic dispersion relation is obtained from: $\nabla_{\perp}^{2} A_{\|}=\mathrm{i} \omega /\left(k_{\|}^{2} \lambda_{\mathrm{De}}^{2} c\right)\left[1+\alpha Z_{0}(\alpha)\right] E_{\|}, \rho_{\mathrm{s}}^{2} \nabla_{\perp}^{2} \phi=$ $\mathrm{i} / k_{\|}\left[1+\alpha Z_{0}(\alpha)\right] E_{\|}$and $E_{\|}=-\mathrm{i} k_{\|} \phi+i \omega A_{\|} / c$. It is

$$
\rho_{\mathrm{s}}^{2} k_{\perp}^{2}+\left(1-\frac{\omega^{2}}{k_{\|}^{2} v_{\mathrm{A}}}\right)\left[1+\alpha Z_{0}(\alpha)\right]=0 .
$$

This is the general complex dispersion relation for the dispersive Alfven wave. In the limit $\alpha \ll 1$, it reads as

$$
\omega^{2}=k_{\|}^{2} v_{\mathrm{A}}^{2}\left[1+k_{\perp}^{2} \rho_{\mathrm{s}}^{2}(1-\mathrm{i} \sqrt{\pi} \alpha)\right] .
$$

Its real part corresponds to the frequency of the kinetic Alfven wave, which was also derived from fluid theory above. Its imaginary part, which corresponds to the Landau damping rate (see also Eq. (28)), reads as

$$
\gamma(\boldsymbol{k})=\frac{\sqrt{\pi}}{2} \frac{v_{\mathrm{A}}^{2}}{v_{\mathrm{te}}} k_{\|} k_{\perp}^{2} \rho_{\mathrm{s}}^{2} .
$$

Most calculations above were finalized in the limit $\alpha \ll 1$. In the opposite limit of $\alpha \gg 1$, one obtains the frequency and damping rate of the inertial Alfven wave, which has its parallel electric field balanced by the electron inertia in Ohm's law. For frequency $\omega \sim k_{\|} v_{\mathrm{A}}, \alpha \sim v_{\mathrm{A}} / v_{\text {te }}$. It means that the kinetic Alfven wave regime corresponds to $v_{\mathrm{A}} / v_{\text {te }} \ll 1$ and the inertial Alfven wave regime corresponds to $v_{\mathrm{A}} / v_{\mathrm{te}} \gg 1$. In the following we continue to focus on the warm plasma regime corresponding to $1 \gg \beta \gg m_{\mathrm{e}} / m_{\mathrm{i}}$, where $\beta$ is the pressure parameter. Landau damping of the inertial Alfven wave and its effect on phase mixing can be treated similarly.

\section{Phase mixing}

Phase mixing of a shear Alfven wave packet can be considered in the framework of an eikonal description:

$$
\begin{aligned}
& \frac{\mathrm{d} \boldsymbol{x}}{\mathrm{d} t}=\nabla_{k} \omega, \\
& \frac{\mathrm{d} \boldsymbol{k}}{\mathrm{d} t}=-\nabla_{x} \omega,
\end{aligned}
$$

with $\omega= \pm k_{\|} v_{\mathrm{A}}$. These are the characteristics of the wave-kinetic equation

$$
\frac{\partial e_{ \pm}}{\partial t}+\nabla_{k} \omega \nabla_{x} e_{ \pm}-\nabla_{x} \omega \nabla_{k} e_{ \pm}=-\gamma(\boldsymbol{k}) e_{ \pm} .
$$

In the latter equation $e_{ \pm}$are the amplitudes of the wave packets corresponding to $\omega= \pm k_{\|} v_{\mathrm{A}}$, and $\gamma(\boldsymbol{k})$ is a wave number dependent damping rate. Following the trajectory of a wave packet in phase space $(\boldsymbol{x}, \boldsymbol{k})$, its amplitude evolves according to

$$
\frac{\mathrm{d} e_{ \pm}}{\mathrm{d} t}=-\gamma(\boldsymbol{k}) e_{ \pm}
$$


This equation is integrated to give

$$
e_{ \pm}(t)=e_{ \pm}(0) \exp \left(-\int \gamma(\boldsymbol{k}) \mathrm{d} t\right) .
$$

The principle of phase mixing is simple: for any damping rate $\gamma$ that is an increasing function of $k$, any mechanism producing an increase in $k$ as a function of time also results in a smaller damping time scale. This is precisely the situation which occurs when the Alfven wave packet propagates along field lines with a transverse variation of the Alfven speed: the wave packet is sheared. In this case, say $\boldsymbol{v}_{\mathrm{A}}(x)=-v_{\mathrm{A}}^{\prime} x \boldsymbol{z}, z$ is the unit vector in the parallel direction and $x$ the transverse coordinate, then

$$
\frac{\mathrm{d} k_{\perp}}{\mathrm{d} t}=k_{\|} v_{\mathrm{A}}^{\prime},
$$

with by definition $v_{\mathrm{A}}^{\prime}=v_{\mathrm{A}} / L_{\perp}, L_{\perp}$ being the characteristic length of the transverse inhomogeneity and $k_{\|}=k_{\|}(t=0)$. This means that $k_{\perp}$ increases linearly with time due to differential advection of the wave packets along the field lines, i.e.,

$$
k_{\perp}(t)=k_{\|} v_{\mathrm{A}}^{\prime} t,
$$

where we have taken $k_{\perp}(t=0)=0$ without loss of generality.

For resistive MHD, Ohm's law reads as $E_{\|}=\eta J_{\|}$and the following results are well known. The damping rate is $\gamma(\boldsymbol{k})=$ $\eta c\left(k_{\perp}^{2}+k_{\|}^{2}\right) / 4 \pi=D_{m}\left(k_{\perp}^{2}+k_{\|}^{2}\right)$. This is the Fourier transform of the operator responsible for magnetic diffusion in the induction equation. Hence,

$$
e_{ \pm}(t)=e_{ \pm}(0) \exp \left[-D_{m} k_{\|}^{2} \int\left(1+v_{\mathrm{A}}^{2} t^{2}\right) \mathrm{d} t\right],
$$

which in the limit $t \gg v_{\mathrm{A}}^{\prime-1}$ yields

$$
e_{ \pm}(t) \sim e_{ \pm}(0) \exp \left(-\frac{D_{m} v_{\mathrm{A}}^{2} k_{\|}^{2}}{3} t^{3}\right) .
$$

Since, $z=v_{\mathrm{A}} t$, we also have

$$
e_{ \pm}(z) \sim e_{ \pm}(0) \exp \left(-\frac{D_{m} v_{\mathrm{A}}^{2} \omega^{2}}{3 v_{\mathrm{A}}^{5}} z^{3}\right),
$$

for an Alfven wave excited at $z=0$ with frequency $\omega$. In a collisionless plasma, when the dissipation is provided by electron Landau damping, with damping rate $\gamma(\boldsymbol{k})=\sqrt{\pi} v_{\mathrm{A}}^{2} k_{\|} k_{\perp}^{2} \rho_{\mathrm{s}}^{2} / 2 v_{\mathrm{te}}$, the equivalent expressions are

$$
e_{ \pm}(t)=e_{ \pm}(0) \exp \left(-\frac{\sqrt{\pi}}{6} \frac{v_{\mathrm{A}}^{2} v_{\mathrm{A}}^{\prime 2}}{v_{\mathrm{te}}} \rho_{\mathrm{s}}^{2} k_{\|}^{3} t^{3}\right)
$$

and

$$
e_{ \pm}(z)=e_{ \pm}(0) \exp \left(-\frac{\sqrt{\pi}}{6} \frac{v_{\mathrm{A}}^{\prime 2}}{v_{\mathrm{A}}^{4} v_{\mathrm{te}}} \rho_{\mathrm{s}}^{2} \omega^{3} z^{3}\right),
$$

for an Alfven wave excited at $z=0$ with frequency $\omega$. The collisionless phase mixing time scale is thus

$$
\tau_{\mathrm{pm}} \sim \frac{v_{\mathrm{te}}^{1 / 3} L_{\perp}^{2 / 3}}{v_{\mathrm{A}}^{4 / 3} \rho_{\mathrm{s}}^{2 / 3} k_{\|}},
$$

and the phase mixing length scale is

$$
l_{\mathrm{pm}} \sim \frac{v_{\mathrm{A}}^{2 / 3} v_{\mathrm{te}}^{1 / 3} L_{\perp}^{2 / 3}}{\rho_{\mathrm{s}}^{2 / 3} \omega} .
$$

The scaling of the phase mixing length scale with the frequency $\omega$ in the spatial problem is different from that of resistive MHD phase mixing since the collisionless conductivity associated with electron Landau damping depends on $\omega$, contrary to Spitzer conductivity. However, the dependence with time or distance of the decay law, like $\exp \left(-\alpha_{1} t^{3}\right)$ or $\exp \left(-\alpha_{2} z^{3}\right)$ is similar to resistive MHD phase mixing. The physical reason is obviously the common scaling of the damping rate $\gamma(\boldsymbol{k})$ with $k_{\perp}$ in the collisional and collisionless cases.

The effect of electron Landau damping on phase mixing was first considered by Voitenko \& Goossens (2000a). They derived a relation identical to Eq. (45) (see Eqs. (30) and (11) in Voitenko $\&$ Goossens 2000a). Moreover, results of the particles-in-cell (PIC) simulations carried by Tsiklauri \& Haruki (2008) have produced $l_{\mathrm{pm}} \propto \omega^{-\zeta}$ with $\zeta \simeq 1.10$, for the dependence of the phase mixing length scale $l_{\text {pm }}$ with frequency $\omega$. They also report that the parallel electric field associated with the Alfven wave is primarily balanced by the electron pressure gradient in their simulations. They attribute the scaling of $l_{\mathrm{pm}}$ with $\omega$ to the effect of an anomalous resistivity. Here, we emphasize that the PIC simulation results can be clearly interpreted as the standard effect of electron Landau damping of the KAW by resonant interaction with electrons since it gives $l_{\mathrm{pm}} \propto \omega^{-\zeta}$ with $\zeta=1$. As a rule, the Landau damping rate is an increasing function of frequency and, for kinetic Alfven waves, we have $\gamma_{\mathrm{L}}\left(k_{\perp}, \omega\right) \sim k_{\perp}^{2} \rho_{\mathrm{S}}^{2}\left(v_{\mathrm{A}} / v_{\mathrm{te}}\right) \omega$. In contrast, the resistive damping rate is independent of frequency and is rewritten as $\gamma_{r}\left(k_{\perp}\right) \sim \mu_{\mathrm{e}} d_{\mathrm{e}}^{2} k_{\perp}^{2}$, where $\mu_{\mathrm{e}}$ is the electron collisional frequency and $d_{\mathrm{e}}=c / \omega_{\mathrm{pe}}$ the electron skin depth. Therefore, it is clear that Landau damping is the dominant damping mechanism only for high-frequency Alfven waves, where $\omega \gg \mu_{\mathrm{e}} \beta_{\mathrm{e}}^{-3 / 2}, \beta_{\mathrm{e}}=v_{\text {te }}^{2} / v_{\mathrm{a}}^{2}$ is the electron pressure parameter. For typical coronal holes conditions, $v_{\mathrm{A}} / v_{\mathrm{te}} \sim 1 / 2$ and $\mu_{\mathrm{e}} \sim 4 \mathrm{~s}^{-1}$, Landau damping dominates resistivity for frequencies higher than $\sim 1 \mathrm{~s}^{-1}$, and such high-frequency Alfven waves can propagate a distance $l \sim 2 \times 10^{5}\left(\mathrm{~km}^{1 / 3} \mathrm{~s}^{-1}\right) \omega^{-1} L_{\perp}^{2 / 3}$.

\section{Parallel electric field generation}

For an Alfven wave created by a source through perturbation of the background magnetic field, a parallel electric field is produced, provided $k_{\perp}$ is finite, which is given by Eq. (16):

$$
E_{\|}=\frac{v_{\mathrm{A}}}{c} k_{\|} \frac{k_{\perp} \rho_{\mathrm{s}}^{2}}{\sqrt{1+k_{\perp}^{2} \rho_{\mathrm{s}}^{2}}} B_{\perp} .
$$

It is this parallel electric field that is responsible for the Landau damping of the wave (see above). For a given $k_{\|}$and $\delta B_{\perp}$, this electric field is amplified provided that the $k_{\perp}$ associated with the wave field is also amplified. The reason is that $E_{\|}$is a monotonic increasing function of $k_{\perp}$. However, $E_{\|}\left(k_{\perp}\right)$ also reaches a plateau for $k_{\perp} \rho_{\mathrm{S}} \sim 1$, which is the boundary between the MHD and the dispersive regime. Indeed,

$$
E_{\|}=\frac{v_{\mathrm{A}}}{c} k_{\|} k_{\perp} \rho_{\mathrm{s}}^{2} B_{\perp}
$$

for $k_{\perp} \rho_{\mathrm{S}} \ll 1$ and $E_{\|}$reaches its maximum, of the order of

$$
E_{\|}=\frac{v_{\mathrm{A}}}{c} k_{\|} \rho_{\mathrm{s}} B_{\perp}
$$

when $k_{\perp} \rho_{\mathrm{s}} \sim 1$ or larger. Therefore, significant amplification of this parallel electric field can only occur in the range of wave numbers where the wave is non-dispersive; i.e., it behaves as a shear-Alfven wave with frequency $\omega \simeq \pm k_{\|} v_{\mathrm{A}}$. 
From the results of the previous section we obtain the dependence with time of the parallel electric field strength during the phase mixing process:

$$
\widetilde{E}_{\|}(t)=v_{\mathrm{A}}^{\prime} k_{\|}^{2} \rho_{\mathrm{s}}^{2} t \exp \left(-\frac{\sqrt{\pi}}{6} \frac{v_{\mathrm{A}}^{2} v_{\mathrm{A}}^{\prime 2}}{v_{\text {te }}} \rho_{\mathrm{s}}^{2} k_{\|}^{3} t^{3}\right),
$$

where a normalized electric field $\widetilde{E}_{\|}=E_{\|} /\left(B_{\perp}(0) v_{\mathrm{A}} / c\right)$ has been defined. The variation with time $\widetilde{E}(t)$ takes the form $\beta_{1} t \exp \left(-\alpha_{1} t^{3}\right)$, with a growth phase followed by a decay phase typical of the alternating field aligned current during phase mixing. Since $z=v_{\mathrm{A}} t$, then

$$
\widetilde{E}_{\|}(z)=\frac{v_{\mathrm{A}}^{\prime} \omega^{2} \rho_{\mathrm{s}}^{2} z}{v_{\mathrm{A}}^{3}} \exp \left(-\frac{\sqrt{\pi}}{6} \frac{v_{\mathrm{A}}^{\prime 2}}{v_{\mathrm{A}}^{4} v_{\mathrm{te}}} \rho_{\mathrm{s}}^{2} \omega^{3} z^{3}\right),
$$

which has the form $\beta_{2} z \exp \left(-\alpha_{2} z^{3}\right)$, for an Alfven wave excited at $z=0$ with frequency $\omega$. The above defined phase mixing time/length scales are precisely those scales associated with the amplification of the parallel electric field; i.e., the time/length scales for the parallel electric field to reach its maximum value given by

$$
\widetilde{E} \sim \frac{\omega v_{\mathrm{te}}^{1 / 3} \rho_{\mathrm{s}}^{4 / 3}}{v_{\mathrm{A}}^{4 / 3} L^{1 / 3}}
$$

with $\omega \simeq k_{\|} v_{\mathrm{A}}$.

\section{Conclusions}

Previous PIC simulations of collisonless phase mixing of Alfven waves (Tsiklauri et al. 2005a,b; Tsiklauri \& Haruki 2008) have identified its relation to the generation of a parallel electric field and acceleration of electrons. The importance of this parallel electric field has been first pointed out by Hasegawa and Chen in the context of resonant absorption. They also show that the dominant energy dissipation of the Alfven wave, in a collisionless low- $\beta$ plasma, involves energy transfer to the electrons (Hasegawa \& Chen 1976). The role of electron Landau damping in collisonless phase mixing has also been considered by Voitenko \& Goossens (2000a,b)

Focusing on the kinetic regime of the dispersive Alfven wave, when $v_{\mathrm{A}} / v_{\mathrm{te}} \ll 1$, we provided a detailed discussion of the role played by phase mixing in both parallel electric field amplification and enhanced electron Landau damping of the wave.

Qualitatively, the physics of collisionless phase mixing can be summarized as follows. A parallel electric field accompanies the propagation of Alfven waves with finite $k_{\perp}$. The magnitude of this electric field is an increasing function of $k_{\perp}$ that saturates in the dispersive range when $k_{\perp} \rho_{\mathrm{S}} \sim 1$ or larger. Therefore, any mechanism that produces an increase in $k_{\perp}$ also leads to the amplification of the parallel electric field associated with the Alfven wave. Phase mixing is such a mechanism, independently of the energy dissipation channel. Phase mixing is a special occurrence of energy-conserving cascade (Bian \& Tsiklauri 2008). Such a cascade, predominantly involving perpendicular wave numbers, can also be produced by non-linear interactions (i.e. turbulence), leading to parallel electric field amplification (Bian \& Kontar 2010; Bian et al. 2010). Existence of this parallel electric field and the dependence of its magnitude with $k_{\perp}$ yield a Landau damping rate, which scales like $k_{\perp}^{2}$, just as visco-resistive damping. This can be demonstrated very simply in the framework of drift-kinetic theory. Therefore, in a collisionless plasma, phase mixing leads to enhanced electron Landau damping of the Alfven wave in a manner that is very similar to the well-studied case of enhanced visco-resistive damping. As a consequence, once the wave has damped in a collisionless low- $\beta$ plasma, its energy has been transferred to the electrons. The time and length scales involved in the damping process were evaluated for small amplitude perturbations. Moreover, we studied the evolution of the magnitude of the parallel electric field in the course of its amplification and calculated its maximum value.

We argued that the scaling of the phase mixing length scale with frequency, $l_{\mathrm{pm}} \propto \omega^{-\zeta}$ and $\zeta \simeq 1$, reported by (Tsiklauri $\&$ Haruki 2008) has a simple interpretation in term of electron Landau damping. PIC simulations of collisionless phase mixing are valuable tools because they can provide direct information on the modification of the electron distribution function involved in the acceleration process, see (Tsiklauri et al. 2005a,b), a feature which the present kind of preliminary analysis is not capable of.

Acknowledgements. This work is supported by an STFC rolling grant (N.H.B., E.P.K.) and an STFC Advanced Fellowship (E.P.K.). Financial support by the Leverhulme Trust grant (F/00179/AY) and by the European Commission through the SOLAIRE Network (MTRN-CT-2006-035484) is gratefully acknowledged.

\section{References}

Bian, N. H., \& Kontar, E. P. 2010, Phys. Plasmas, 17, 062308

Bian, N., \& Tsiklauri, D. 2008, A\&A, 489, 1291

Bian, N. H., \& Tsiklauri, D. 2009, Phys. Plasmas, 16, 064503

Bian, N. H., Kontar, E. P., \& Brown, J. C. 2010, A\&A, 519, A114

Botha, G. J. J., Arber, T. D., Nakariakov, V. M., \& Keenan, F. P. 2000, A\&A, 363,1186

De Moortel, I., Hood, A. W., \& Arber, T. D. 2000, A\&A, 354, 334

Goertz, C. K., \& Boswell, R. W. 1979, J. Geophys. Res., 84, 7239

Hasegawa, A. 1976, J. Geophys. Res., 81, 5083

Hasegawa, A. 1985, in Unstable Current Systems and Plasma Instabilities in Astrophysics, ed. M. R. Kundu, \& G. D. Holman, IAU Symp., 107, 381

Hasegawa, A., \& Chen, L. 1974, Phys. Rev. Lett., 32, 454

Hasegawa, A., \& Chen, L. 1976, Phys. Fluids, 19, 1924

Hasegawa, A., \& Mima, K. 1978, J. Geophys. Res., 83, 1117

Heyvaerts, J., \& Priest, E. R. 1983, A\&A, 117, 220

Hood, A. W., Brooks, S. J., \& Wright, A. N. 2002, Roy. Soc. Lond. Proc. Ser. A, 458,2307

Ionson, J. A. 1978, ApJ, 226, 650

Nakariakov, V. M., Roberts, B., \& Murawski, K. 1997, Sol. Phys., 175, 93

Parker, E. N. 1991, ApJ, 376, 355

Schekochihin, A. A., Cowley, S. C., Dorland, W., et al. 2009, ApJS, 182, 310

Tsiklauri, D., \& Haruki, T. 2008, Phys. Plasmas, 15, 112902

Tsiklauri, D., Sakai, J., \& Saito, S. 2005a, A\&A, 435, 1105

Tsiklauri, D., Sakai, J., \& Saito, S. 2005b, New J. Phys., 7, 79

Voitenko, Y., \& Goossens, M. 2000a, A\&A, 357, 1086

Voitenko, Y., \& Goossens, M. 2000b, A\&A, 357, 1073 